\title{
The Rhinologist and the ENT Practitioner
}

Twenty years ago, rhinology was a minor part of general ENT and not necessarily the most attractive one. The widest interest was raised by otology and head and neck surgery. However, some audacious individuals were interested in the physiology and pathology of the nose and sinuses. During a period of almost 20 years and with enthousiasm, they have created a new field of interest, developed better knowledge of the system, new diagnostic tools and more efficient therapies. Today, rhinology is considered as a specific entity. It has become an independent division in most ENT centers with specific training programs. It has led to the creation of new journals as well as to the organization of regular national and international meetings solely devoted to this topic.

The existence of divisions of rhinology in university hospitals is necessary to guarantee its further research and development. However, arguments in favor of rhinology as a specific specialization independent of general ENT are lacking. In private practice, rhinology is today an important part of the activity of general ENT practitioners. Therefore, continuous teaching of general practitioners is also an important mission of researchers.

In preparing this special issue of Otorhinolaryngologia Nova, the editors wanted to help the readers of the Journal to better understand the most recent developments and questions concerning etiologic considerations of chronic sinusitis and its therapeutic consequences. Prominent experts were invited to express their views on this fast evolving field. When the project was launched, several experts accepted immediately to participate in the debate. Unfortunately, and unexpectedly, some changed their mind and declined the invitation after the deadline for the submission of their manuscript. As it was already too late to restart the whole project with other experts, it has been decided to publish the works of those colleagues who had delivered a paper. We want to thank them warmly for their contribution. It is expected that some of the opinions expressed in this issue will raise additional comments among readers, which we will be pleased to publish in the next issue of Otorhinolaryngologia Nova, keeping the debate opened. 